\title{
Minireview
}

\section{mTOR signaling: implications for cancer and anticancer therapy}

\author{
E Petroulakis ${ }^{1,2}$, Y Mamane ${ }^{1,2}$, O Le Bacquer', D Shahbazian' and N Sonenberg,,1 \\ 'Department of Biochemistry, McGill Cancer Centre, McGill University, 3655 Promenade Sir William Osler, Montreal, QUE, Canada H3G I Y6
}

\begin{abstract}
Mounting evidence links deregulated protein synthesis to tumorigenesis via the translation initiation factor complex elF4F. Components of this complex are often overexpressed in a large number of cancers and promote malignant transformation in experimental systems. mTOR affects the activity of the elF4F complex by phosphorylating repressors of the elF4F complex, the elF4E binding proteins. The immunosuppressant rapamycin specifically inhibits mTOR activity and retards cancer growth. Importantly, mutations in upstream negative regulators of mTOR cause hamartomas, haemangiomas, and cancers that are sensitive to rapamycin treatment. Such mutations lead to increased elF4F formation and consequently to enhanced translation initiation and cell growth. Thus, inhibition of translation initiation through targeting the mTOR-signalling pathway is emerging as a promising therapeutic option. British Journal of Cancer (2006) 94, 195-199. doi: I 0.1038/sj.bjc.6602902 www.bjcancer.com
\end{abstract}

Published online 13 December 2005

c) 2006 Cancer Research UK

Keywords: translational control; elF4F; elF4E binding proteins; rapamycin; mTOR; malignant transformation

Rapamycin (Rapamune ${ }^{\circledR}$, Wyeth Ayerst) is a specific inhibitor of the target of rapamycin (TOR) (for review see Hay and Sonenberg, 2004). The protein kinase TOR genes (TOR1 and TOR2) were first identified in the early 1990s in a screen for rapamycin-resistant yeast mutants (Heitman et al, 1991). The mammalian orthologue (mTOR), is also referred to as FKBP12-rapamycin associated protein (FRAP), rapamycin and FKBי12 target (RAFT), rapamycin target (RAPT), or sirolimus effector protein (SEP). Rapamycin forms a complex with the immunophilin FK506 binding protein-12 (FKBP12), which binds to the FKBP12-rapamycin binding (FRB) domain of mTOR and inhibits its kinase activity. Rapamycin inhibits the growth of a broad spectrum of cancers including rhabdomyosarcoma, neuroblastoma, glioblastoma, small cell lung carcinoma, osteosarcoma, pancreatic cancer, leukaemia, B-cell lymphoma, and breast and colon cancer-derived cells (Huang and Houghton, 2002). The rapamycin analogues, CCI-779 (WyethAyerst, PA, USA), RAD001 (Novartis, Switzerland), and AP23573 (Ariad Pharmaceuticals, MA, USA) have shown promise in clinical trials (Panwalkar et al, 2004).

mTOR functions by integrating extracellular signals (growth factors and hormones), with amino-acid availability and intracellular energy status to control translation rates and additional metabolic processes (Hay and Sonenberg, 2004). mTOR enhances translation initiation in part by phosphorylating two major targets, the eIF4E binding proteins (4E-BPs) and the ribosomal protein S6 kinases ( $\mathrm{S} 6 \mathrm{~K} 1$ and $\mathrm{S} 6 \mathrm{~K} 2$ ) that cooperate to regulate translation initiation rates.

\footnotetext{
*Correspondence: Dr N Sonenberg;

E-mail: nahum.sonenberg@mcgill.ca

${ }^{2}$ These authors contributed equally to this article.

Received 12 August 2005; revised 10 November 2005; accepted 14 November 2005; published online 13 December 2005
}

Translation initiation is implicated in tumorigenesis All nuclear transcribed eukaryotic mRNAs contain a 'cap' structure, $\mathrm{m}^{7} \mathrm{GpppN}$ (where ' $N$ ' is any nucleotide and ' $m$ ' is a methyl group), at the $5^{\prime}$ terminus. The 'cap' is specifically bound by the initiation factor eIF4E that associates with two additional initiation factors, eIF4G (a large scaffolding protein) and eIF4A (a helicase which is believed to unwind mRNA $5^{\prime}$ secondary structures), to form the eIF4F complex, which facilitates the recruitment of ribosomes to the mRNA (Gingras et al, 1999).

Several studies demonstrated that eIF4E acts as an oncogene (for a recent review see Mamane et al, 2004). eIF4E levels are limiting for cap-dependent translation in most systems and overexpression of eIF4E in rodent cells causes malignant transformation (LazarisKaratzas et al, 1990; Mamane et al, 2004). Therefore, increased eIF4E expression in cancer cells is thought to enhance eIF4F complex formation and as a consequence, the translation of a subset of mRNAs that contain highly structured $5^{\prime}$ untranslated regions (UTRs), such as vascular endothelial growth factor (VEGF) and ornithine decarboxylase (ODC). Indeed, eIF4E is elevated in numerous types of cancers, including bronchioalveolar, bladder, head and neck, liver, colon, and breast cancers (De Benedetti and Graff, 2004). Consistent with this, two important studies demonstrated that overexpression of eIF4E in mice promotes lymphomagenesis (Ruggero et al, 2004; Wendel et al, 2004). Furthermore, the importance of eIF4E's role in tumorigenesis is reinforced by the finding that eIF4F complex is necessary for maintaining tumour cell growth (Avdulov et al, 2004). Importantly, siRNA treatment to reduce eIF4E expression inhibits the growth of several cell lines including those of head and neck squamous carcinoma cells (Oridate et al, 2005).

Other components of the eIF4F complex are also implicated in malignant transformation. eIF4GI can transform NIH 3T3 cells, and is overexpressed in squamous cell lung carcinomas and breast cancer cell lines (see Mamane et al (2004)). eIF4A is also overexpressed in human melanoma cell lines and in primary 
hepatocellular carcinomas (see Mamane et al (2004)). Hence, these findings reinforce a molecular model where increased eIF4F complex promotes tumorigenesis.

Rapamycin inhibits cap-dependent translation Growth inhibition by rapamycin is thought to be partially mediated by the inhibition of cap-dependent translation (Hay and Sonenberg, 2004). The mammalian 4E-BPs constitute a three-member family of translational inhibitors that bind to eIF4E, inhibit formation of the eIF4F complex and thus cap-dependent translation (Gingras et al, 1999). The $4 \mathrm{E}$-BPs are dephosphorylated as a consequence of rapamycin treatment. Binding of $4 \mathrm{E}-\mathrm{BP} 1$ (the best studied 4E-BP family member) to eIF4E is regulated by its phosphorylation status: the hypophosphorylated form (residues Thr37 and 46) of 4E-BP1 binds very tightly to eIF4E and inhibits cap-dependent translation. In response to stimuli such as growth factors, hormones, nutrients, or increased energy levels, mTOR phosphorylates 4E-BP1 primarily at residues Ser65 and Thr73. As a result, eIF4E is released from 4EBP1 with a subsequent increase in cap-dependent translation (Mamane et al, 2004).

Several studies addressed the role of the 4E-BPs in tumour suppression. For example, 4E-BP overexpression counteracts eIF4E-, src-, or ras-mediated cellular transformation (Mamane et al, 2004). Also, a nonphosphorylatable mutant of 4E-BP1, which constitutively binds eIF4E, inhibits breast cancer cell growth more strongly than wild-type 4E-BP1 (Avdulov et al, 2004). In addition, the $4 \mathrm{E}-\mathrm{BPs}$ appear to mediate rapamycin-sensitivity since rhabdomyosarcoma cells expressing very low levels of 4E-BP1 are refractory to growth inhibition by rapamycin (Dilling et al, 2002). Thus, the $4 \mathrm{E}-\mathrm{BPs}$ are considered to be important mTOR targets that modulate cancer cell growth through a mechanism that involves cap-dependent translation.

Ribosomal protein S6 kinase The ribosomal protein S6 kinases are also important substrates of mTOR. S6K1 (p70S6K; the better characterised S6 kinase) is implicated in the positive regulation of cell growth and proliferation (Fumagalli and Thomas, 2000). Interestingly, S6K1 activation correlates with enhanced translation of a subset of mRNAs that contain a terminal $5^{\prime}$ oligopyrimidine tract (TOP mRNAs) (Fumagalli and Thomas, 2000). These mRNAs encode ribosomal proteins, elongation factors, the poly-A binding protein and other components of the translational machinery that become selectively translated in response to growth factors. However, TOP mRNA translation remains intact even when both S6K1 and S6K2 genes are genetically disrupted in mice (Pende et al, 2004), indicating that S6Ks are not essential for the regulation of TOP mRNA translation.

S6K regulates mTOR through a negative feedback signalling pathway that affects insulin receptor substrate-1 (IRS-1) (Figure 1). S6K was shown to directly phosphorylate IRS-1 to inhibit phospohatidylinositol-3-kinase (PI3K) and Akt activation (Harrington et al, 2004). S6K activation decreases IRS-1 expression, while rapamycin treatment restores IRS-1 expression (Harrington et al, 2004). Also, deletion of S6K1 in mice renders them resistant to age- and high-fat diet-induced obesity while enhancing insulin sensitivity (Um et al, 2004). Lastly, Akt activation in response to insulin is enhanced in S6K-null mice, thus implicating S6K1 in diabetes and obesity (Um et al, 2004).

Other translational targets of $m$ TOR Other rapamycin-sensitive targets involved in translational regulation exist. The translation initiation factor eIF4B, which stimulates the activity of eIF4A in unwinding duplex RNA, is phosphorylated by S6K1 on serine 422 (Raught et al, 2004). mTOR also mediates the phosphorylation of serines 1108, 1148, and 1192 in the C-terminus of eIF4G (Raught et al, 2000). In addition, mTOR regulates the elongation stage of protein synthesis by modulating elongation factor (eEF2) kinase (Proud, 2004).

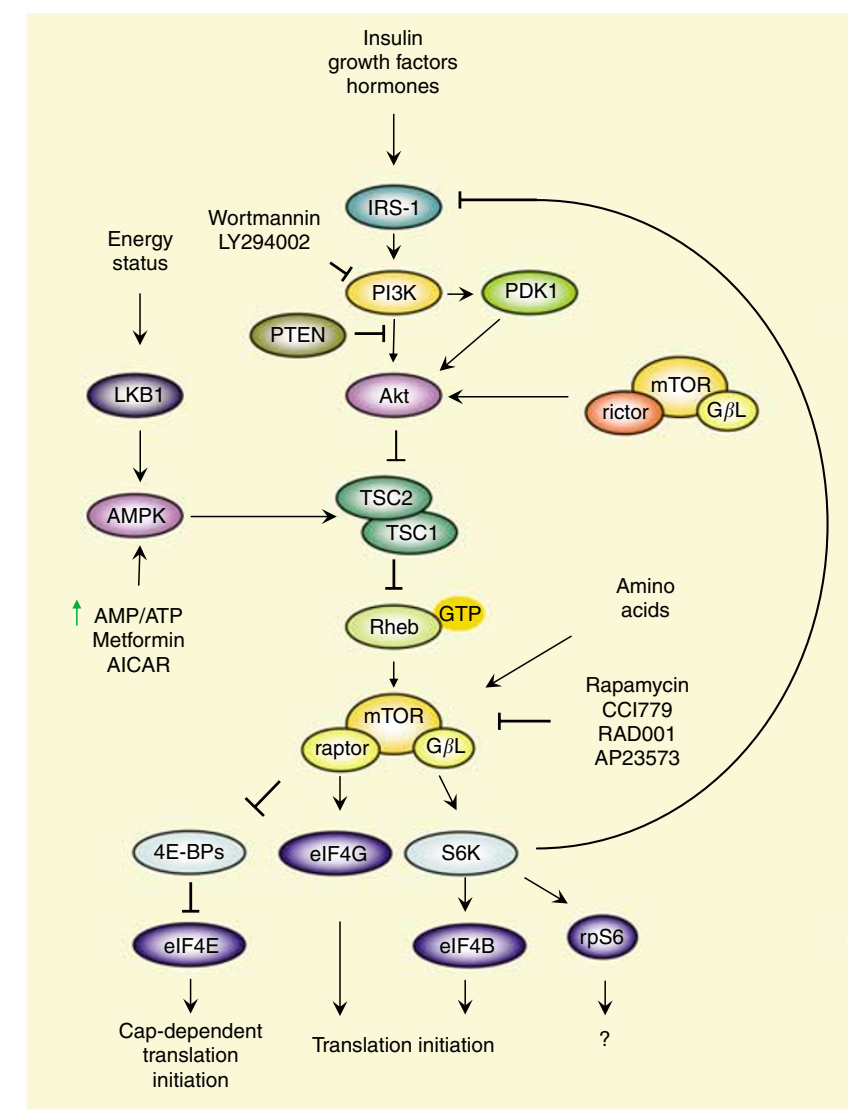

Figure I mTOR signaling to translation initiation. mTOR signaling regulates translation initiation by integrating several different inputs. Insulin, hormones and growth factors activate the PI3K/Akt signaling cascade. Akt receives inputs from $\mathrm{PDK}$ and the rictor/G $\beta \mathrm{L} / \mathrm{mTOR}$ complex. Energy status (the AMP/ATP ratio) modulates AMPK activity. These pathways affect TSCI/2 to regulate $\mathrm{mTOR}$ activity via Rheb. The raptor/G $\beta \mathrm{L} / \mathrm{mTOR}$ complex mediates the phosphorylation of 4E-BP and S6K. Pharmacological inhibition of PI3K (wortmannin or LY294002) and mTOR (rapamycin or its analogs), or activation of AMPK (by increased AMP/ATP, metformin or AICAR) are indicated.

PI3K/Akt/mTOR signaling Growth factors, mitogens and hormones activate the PI3K signaling pathway and consequently mTOR (Hay and Sonenberg, 2004) (Figure 1). Nutrients (amino acids, glucose) also regulate mTOR activity to affect translation (Proud, 2004). PI3K phosphorylates phosphatidylinositol-4,5-bisphosphate (PIP2) to generate phosphatidylinositol-3,4,5-triphosphate (PIP3). PIP3 binds and activates pleckstrin homology $(\mathrm{PH})$ domain-bearing proteins such as Akt and the phosphoinositide dependent kinase (PDK) family members. PDK1 and the Ser/ Thr kinase Akt are recruited to the membrane where PDK1 phosphorylates and activates Akt. In turn, Akt phosphorylates the tuberous sclerosis complex, TSC1/TSC2 (hamartin/tuberin) that serves as a GTPase activating protein (GAP) for the small G protein, Ras homolog enriched in brain (Rheb). Rheb, in its GTPbound state, can activate mTOR.

\section{Negative regulators of $\mathrm{mTOR}$ are implicated in tumour suppression}

PTEN PTEN is a lipid phosphatase that acts on the lipid substrate PIP3 to convert it to PIP2. PIP3 accumulation results from PTENinactivating mutations to effect cell size, adhesion, motility, and 
angiogenesis (Inoki et al, 2005a). PTEN is frequently mutated in many cancers and in a group of cancer-like syndromes including Cowden, Lhermitte-Duclos, Bannayan-Zonana, and Proteus syndromes that are characterised by the emergence of hamartomas (Inoki et al, 2005a). A critical outcome of PTEN inactivation is an increase in mTOR activity, resulting in the phosphorylation of $4 \mathrm{E}-$ BPs and S6Ks. Strikingly, PTEN-I- cells are hypersensitive to growth inhibition by rapamycin and CCI-779 (Guertin and Sabatini, 2005).

AMP-activated protein kinase (AMPK) mTOR activity becomes repressed under conditions of energy deprivation, in which an elevated AMP/ATP intracellular ratio causes activation of AMPK. Stressful conditions, such as nutrient deprivation, hypoxia, heat shock, and ischaemia diminish cellular energy reserves. The link between mTOR inhibition and AMPK was first demonstrated with the use of AICAR (5'-phosphoribosyl-5-aminoimidazole-4-carboxamide ribonucleoside), an AMPK activator, thus linking the amino acid- and energy-sensing functions of mTOR. Metformin, an antidiabetic drug, also activates AMPK and is hypothesised to reduce the risk of cancer in patients with type 2 diabetes (Evans et al, 2005). Thus, targeting AMPK may be an interesting therapeutic option for cancer therapy.

LKB1 LKB1 is a serine/threonine kinase that phosphorylates and activates AMPK. LKB1 is frequently mutated in Peutz-Jeghers syndrome (PJS), an autosomal dominant inherited cancer predisposing patients to the development of tumours mainly throughout the gastrointestinal tract. Disruption of LKB1 in mice causes gastrointestinal and hepatic polyps (Bardeesy et al, 2002). In LKB1-deficient cells, phosphorylation AMPK is defective and cells resist mTOR inactivation in response to AICAR (Shaw et al, 2004). Importantly, 4E-BP1 is less enriched in eIF4E-bound cap analogue precipitates from LKB1-deficient cells (Shaw et al, 2004). This is indicative of enhanced eIF4F formation as a result of increased mTOR signaling caused by LKB1-deficiency. LKB1-mediated inhibition of mTOR also involves the TSC1/2 complex, thus demonstrating the link between LKB1 and mTOR through the regulation of AMPK and TSC1/2 activities (Shaw et al, 2004).

TSC1/TSC2 TSC1 and TSC2 are mutated in tuberous sclerosis complex (TSC) patients. In response to low energy levels, TSC2 becomes phosphorylated by AMPK, resulting in increased stability of the TSC1/2 complex. The stabilised TSC1/2 complex inhibits mTOR and protects cells from energy deprivation-induced apoptosis (see recent reviews by (Inoki et al, 2005b; Kwiatkowski and Manning, 2005)). TSC2 is also phosphorylated by Akt leading to its inactivation and consequently to increased mTOR activity. Furthermore, S6K phosphorylation is elevated in TSC-I- cells and is rapidly abrogated by rapamycin treatment. Activation of the extracellular signal-regulated kinase (Erk) by the Ras signaling pathway also leads to TSC1/2 inactivation through phosphorylation of TSC2 on Ser664 (Ma et al, 2005). Taken together, the TSC1/2 complex functions as a key player in the regulation of the mTOR pathway by receiving inputs from PI3K/PTEN/Akt and Ras signalling pathways, energy levels, and amino acids to regulate translation initiation and affect cell growth and proliferation.

\section{mTOR COMPLEXES REGULATE RAPAMYCIN SENSITIVITY}

Recent findings shed new light on the mechanisms of mTOR sensitivity to rapamycin. Three key interacting partners of mTOR (raptor, G $\beta \mathrm{L}$, and rictor) modulate many of mTOR's rapamycinsensitive and insensitive functions. These evolutionarily conserved proteins have been characterised in yeast and mammalian cells. Raptor is the mammalian ortholog of yeast Kog1 (Kontroller of growth 1) (reviewed in Kim and Sabatini (2004), Lorberg and Hall (2004)). Raptor interacts with mTOR and tethers it to its downstream effectors through a TOS (TOR signaling) motif found in the mTOR substrates, 4E-BP1 and S6K1 (amino acid sequences: FEMDI and FDIDL, respectively) (reviewed in Proud (2004)). The raptor/mTOR interaction is nutrient-sensitive and is dependent on the presence of another partner, $\mathrm{G} \beta \mathrm{L}$ (a mammalian ortholog of yeast Lst8p) (Guertin and Sabatini, 2005). G $\beta \mathrm{L} / \mathrm{mL}$ st8 interacts with the mTOR kinase domain independently of raptor and potentiates mTOR activity. Rictor (rapamycin insensitive component of TOR; the yeast AVO3 ortholog), is involved in the control of cytoskeleton organisation (Lorberg and Hall, 2004; Guertin and Sabatini, 2005). Unlike raptor/G $\beta \mathrm{L} / \mathrm{mTOR}$ (mTORC1 complex), the rictor/mTOR (mTORC2 complex) interaction is insensitive to rapamycin treatment. Most importantly, the rictor/mTOR complex is required for Akt phosphorylation on Ser473 to achieve its full activation (Guertin and Sabatini, 2005). Although the activity of the rictor/mTOR complex is insensitive to short periods of rapamycin treatment, it has been suggested that long-term exposure to rapamycin could prevent newly-synthesised mTOR molecules from associating with rictor, thereby preventing rictor/ mTOR-mediated Akt phosphorylation (Guertin and Sabatini, 2005). This model predicts that rictor could serve as a new therapeutic target in mTOR-linked hamartoma syndromes (such as tuberous sclerosis) and PTEN-mutated cancers, where rapamycin therapy has already proven beneficial.

\section{RAPAMYCIN IN COMBINATION THERAPY}

In Peutz Jeghers syndrome, Tuberous Sclerosis, and other diseases where PTEN is inactivated, the use of rapamycin as a clinical means to reverse the effect of elevated mTOR activity is an attractive option (Inoki et al, 2005a). These diseases are distinct from other hamartoma-associated disorders (such as VHL syndrome) since they have an established molecular link to mTOR (Inoki et al, 2005a). Earlier studies demonstrated that PTENinactivated tumour cells exhibit enhanced sensitivity to the rapamycin analog CCI-779 (Guertin and Sabatini, 2005). More recently, several studies have shown that rapamycin treatment, in combination with other chemotherapeutic drugs, can lead to enhanced selective killing of cancer cells. In particular, the protein tyrosine kinase (PTK) inhibitor, Imatinib (Gleevec, STI571) synergises with rapamycin to inhibit BCR/ABL transformed cells (Mohi et al, 2004). The effect of rapamycin may be enhanced as a result of Imatinib-induced Akt/mTOR signaling, a complication that is thought to lead to Imatinib resistance (Burchert et al, 2005). Rapamycin can also synergise with paclitaxel, carboplatin, and vinorelbine to induce apoptosis in breast cancer cells (Mondesire et al, 2004). Cisplatin-induced apoptosis of A549 lung cancer cells is also significantly enhanced when combined with RAD001 (Beuvink et al, 2005). This could be in part due to reduced translation of p53-activated p21 mRNA in A549 and MCF7 cells treated with RAD001, thereby allowing the dosage of cisplatin to be reduced (Beuvink et al, 2005). Also, the use of the EGFR/VEGFR inhibitor, AEE788, in combination with RAD001 greatly decreased tumour growth in glioma xenografts (Goudar et al, 2005). Furthermore, targeting the glycolytic pathway in combination with mTOR inhibition may also be useful in cases where DNAdamaging agents are less efficient in inhibiting growth and promoting apoptosis of cancer cells (Xu et al, 2005).

\section{TARGETING THE TRANSLATIONAL APPARATUS AS A THERAPEUTIC APPROACH}

A critical outcome of mTOR activation is the phosphorylation of several components of the translational apparatus, which mediate translation initiation and cell proliferation. A major rate-limiting 
step in translation initiation is the formation of the eIF4F complex at the $5^{\prime}$ mRNA cap structure. Rapamycin inhibits eIF4F formation largely in part through 4E-BP dephosphorylation. Several studies have implicated the eIF4E/4E-BP pathway as a putative downstream target of mTOR in tumorigenesis (Avdulov et al, 2004; Ruggero et al, 2004; Wendel et al, 2004). Inhibition of mTORmediated cap-dependent translation, through the use of rapamycin is insufficient to elicit a complete inhibition of cancer cell proliferation. This may be in part due to chemoresistance that arises as a consequence of Akt-mediated activation. Hence, the benefits of rapamycin treatment may not be realised in all cases of aberrant mTOR activity. Since experimental models implicate translation initiation directly in cancer cell growth, it would be of interest to develop molecules that specifically target downstream components of the mTOR signaling pathways, which control eIF4F formation.

Recent work has implicated microRNAs (miRNAs) in the regulation of translation initiation by inhibiting cap-dependent translation (Pillai et al, 2005). Importantly, many miRNAs function as oncogenes or tumour suppressors (Chen, 2005). Thus, it is possible that the mTOR pathway could control the

\section{REFERENCES}

Avdulov S, Li S, Michalek V, Burrichter D, Peterson M, Perlman DM, Manivel JC, Sonenberg N, Yee D, Bitterman PB, Polunovsky VA (2004) Activation of translation complex eIF4F is essential for the genesis and maintenance of the malignant phenotype in human mammary epithelial cells. Cancer Cell 5: 553-563

Bardeesy N, Sinha M, Hezel AF, Signoretti S, Hathaway NA, Sharpless NE, Loda M, Carrasco DR, DePinho RA (2002) Loss of the Lkbl tumour suppressor provokes intestinal polyposis but resistance to transformation. Nature 419: $162-167$

Beuvink I, Boulay A, Fumagalli S, Zilbermann F, Ruetz S, O’Reilly T, Natt F, Hall J, Lane HA, Thomas G (2005) The mTOR inhibitor RAD001 sensitizes tumor cells to DNA-damaged induced apoptosis through inhibition of p21 translation. Cell 120: 747-759

Burchert A, Wang Y, Cai D, von Bubnoff N, Paschka P, Muller-Brusselbach S, Ottmann OG, Duyster J, Hochhaus A, Neubauer A (2005) Compensatory $\mathrm{PI} 3$-kinase/Akt/mTor activation regulates imatinib resistance development. Leukemia 19: 1774-1782

Chen CZ (2005) MicroRNAs as oncogenes and tumor suppressors. N Engl J Med 353: $1768-1771$

De Benedetti A, Graff JR (2004) eIF-4E expression and its role in malignancies and metastases. Oncogene 23: 3189-3199

Dilling MB, Germain GS, Dudkin L, Jayaraman AL, Zhang X, Harwood FC, Houghton PJ (2002) 4E-binding proteins, the suppressors of eukaryotic initiation factor $4 \mathrm{E}$, are down-regulated in cells with acquired or intrinsic resistance to rapamycin. J Biol Chem 277: 13907-13917

Evans JM, Donnelly LA, Emslie-Smith AM, Alessi DR, Morris AD (2005) Metformin and reduced risk of cancer in diabetic patients. BMJ 330: $1304-1305$

Fumagalli S, Thomas G (2000) S6 phosphorylation and signal transduction. In Translational Control of Gene Expression, Sonenberg N, Hershey JWB and Mathews MB (eds) pp 695-717. Cold Spring Harbor Laboratory Press: Cold Spring Harbor, New York

Gingras AC, Raught B, Sonenberg N (1999) eIF4 initiation factors: effectors of mRNA recruitment to ribosomes and regulators of translation. Annu Rev Biochem 68: 913-963

Goudar RK, Shi Q, Hjelmeland MD, Keir ST, McLendon RE, Wikstrand CJ, Reese ED, Conrad CA, Traxler P, Lane HA, Reardon DA, Cavenee WK, Wang XF, Bigner DD, Friedman HS, Rich JN (2005) Combination therapy of inhibitors of epidermal growth factor receptor/vascular endothelial growth factor receptor 2 (AEE788) and the mammalian target of rapamycin (RAD001) offers improved glioblastoma tumor growth inhibition. Mol Cancer Ther 4: $101-112$

Guertin DA, Sabatini DM (2005) An expanding role for mTOR in cancer. Trends Mol Med 11: 353-361

Harrington LS, Findlay GM, Gray A, Tolkacheva T, Wigfield S, Rebholz H, Barnett J, Leslie NR, Cheng S, Shepherd PR, Gout I, Downes CP, Lamb RF activity of miRNAs in cancer. It will be important to continue to study the PI3K/Akt/mTOR pathway and its downstream effectors of translation as molecular targets for anticancer therapies.

\section{ACKNOWLEDGEMENTS}

We apologize to those authors whose work is not cited due to editorial space limitations. We thank Colin Lister and Pamela Kirk for their assistance. This research was supported by grants from the National Cancer Institute of Canada (NCIC), the Howard Hughes Medical Institute (HHMI) and Canadian Institutes of Health Research (CIHR) of Canada to NS. EP was supported by a Fellowship from the Cancer Research Society and a Chemical Biology Fellowship from McGill University. YM is a recipient of an NCIC fellowship. DS is supported by a McGill Majors Fellowship from McGill University. OLB is supported by a Chemical Biology Fellowship. NS is a James McGill Professor, a CIHR distinguished scientist and an HHMI International Scholar.
(2004) The TSC1-2 tumor suppressor controls insulin-PI3K signaling via regulation of IRS proteins. J Cell Biol 166: 213-223

Hay N, Sonenberg N (2004) Upstream and downstream of mTOR. Genes Dev 18: 1926 - 1945

Heitman J, Movva NR, Hall MN (1991) Targets for cell cycle arrest by the immunosuppressant rapamycin in yeast. Science 253: $905-909$

Huang S, Houghton PJ (2002) Inhibitors of mammalian target of rapamycin as novel antitumor agents: from bench to clinic. Curr Opin Investig Drugs 3: $295-304$

Inoki K, Corradetti MN, Guan KL (2005a) Dysregulation of the TSC-mTOR pathway in human disease. Nat Genet 37: 19-24

Inoki K, Ouyang H, Li Y, Guan KL (2005b) Signaling by target of rapamycin proteins in cell growth control. Microbiol Mol Biol Rev 69: 79-100

Kim DH, Sabatini DM (2004) Raptor and mTOR: subunits of a nutrientsensitive complex. Curr Top Microbiol Immunol 279: 259-270

Kwiatkowski DJ, Manning BD (2005) Tuberous sclerosis: a GAP at the crossroads of multiple signaling pathways. Hum Mol Genet 14(Suppl 2): R251 - R258

Lazaris-Karatzas A, Montine KS, Sonenberg N (1990) Malignant transformation by a eukaryotic initiation factor subunit that binds to mRNA 5 cap. Nature 345: $544-547$

Lorberg A, Hall MN (2004) TOR: the first 10 years. Curr Top Microbiol Immunol 279: 1 - 18

Ma L, Chen Z, Erdjument-Bromage H, Tempst P, Pandolfi PP (2005) Phosphorylation and functional inactivation of TSC2 by Erk implications for tuberous sclerosis and cancer pathogenesis. Cell 121: 179-193

Mamane Y, Petroulakis E, Rong L, Yoshida K, Ler LW, Sonenberg N (2004) eIF4E - from translation to transformation. Oncogene 23: 3172-3179

Mohi MG, Boulton C, Gu TL, Sternberg DW, Neuberg D, Griffin JD, Gilliland DG, Neel BG (2004) Combination of rapamycin and protein tyrosine kinase (PTK) inhibitors for the treatment of leukemias caused by oncogenic PTKs. Proc Natl Acad Sci USA 101: 3130-3135

Mondesire WH, Jian W, Zhang H, Ensor J, Hung MC, Mills GB, MericBernstam F (2004) Targeting mammalian target of rapamycin synergistically enhances chemotherapy-induced cytotoxicity in breast cancer cells. Clin Cancer Res 10: 7031 - 7042

Oridate N, Kim HJ, Xu X, Lotan R (2005) Growth inhibition of head and neck squamous carcinoma cells by small interfering RNAs targeting eIF4E or cyclin D1 alone or combined with cisplatin. Cancer Biol Ther 4: $318-323$

Panwalkar A, Verstovsek S, Giles FJ (2004) Mammalian target of rapamycin inhibition as therapy for hematologic malignancies. Cancer 100: $657-666$

Pende M, Um SH, Mieulet V, Sticker M, Goss VL, Mestan J, Mueller M, Fumagalli S, Kozma SC, Thomas G (2004) S6K1(-/-)/S6K2(-/-) mice exhibit perinatal lethality and rapamycin-sensitive $5^{\prime}$-terminal 
oligopyrimidine mRNA translation and reveal a mitogen-activated protein kinase-dependent S6 kinase pathway. Mol Cell Biol 24: $3112-3124$

Pillai RS, Bhattacharyya SN, Artus CG, Zoller T, Cougot N, Basyuk E, Bertrand E, Filipowicz W (2005) Inhibition of translational initiation by Let-7 MicroRNA in human cells. Science 309: $1573-1576$

Proud CG (2004) mTOR-mediated regulation of translation factors by amino acids. Biochem Biophys Res Commun 313: 429-436

Raught B, Gingras AC, Gygi SP, Imataka H, Morino S, Gradi A, Aebersold $\mathrm{R}$, Sonenberg N (2000) Serum-stimulated, rapamycin-sensitive phosphorylation sites in the eukaryotic translation initiation factor 4GI. EMBO J 19: $434-444$

Raught B, Peiretti F, Gingras AC, Livingstone M, Shahbazian D, Mayeur GL, Polakiewicz RD, Sonenberg N, Hershey JW (2004) Phosphorylation of eucaryotic translation initiation factor $4 \mathrm{~B}$ Ser422 is modulated by S6 kinases. EMBO J 23: 1761 - 1769
Ruggero D, Montanaro L, Ma L, Xu W, Londei P, Cordon-Cardo C, Pandolfi PP (2004) The translation factor eIF-4E promotes tumor formation and cooperates with c-Myc in lymphomagenesis. Nat Med 10: 484-486

Shaw RJ, Bardeesy N, Manning BD, Lopez L, Kosmatka M, DePinho RA, Cantley LC (2004) The LKB1 tumor suppressor negatively regulates mTOR signaling. Cancer Cell 6: $91-99$

Um SH, Frigerio F, Watanabe M, Picard F, Joaquin M, Sticker M, Fumagalli S, Allegrini PR, Kozma SC, Auwerx J, Thomas G (2004) Absence of S6K1 protects against age- and diet-induced obesity while enhancing insulin sensitivity. Nature 431: 200-205

Wendel HG, De Stanchina E, Fridman JS, Malina A, Ray S, Kogan S, Cordon-Cardo C, Pelletier J, Lowe SW (2004) Survival signalling by Akt and eIF4E in oncogenesis and cancer therapy. Nature 428: $332-337$

Xu RH, Pelicano H, Zhang H, Giles FJ, Keating MJ, Huang P (2005) Synergistic effect of targeting mTOR by rapamycin and depleting ATP by inhibition of glycolysis in lymphoma and leukemia cells. Leukemia 19: $2153-2158$ 